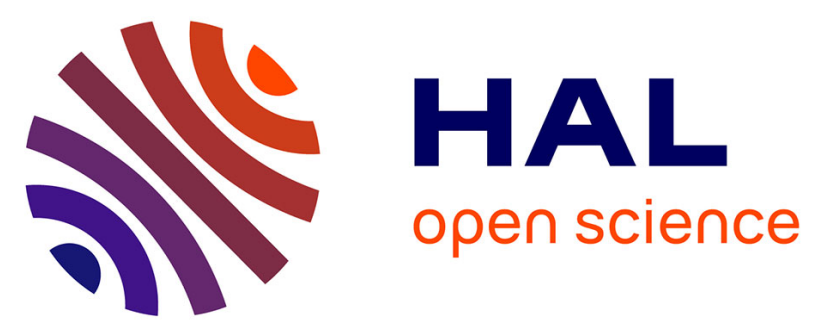

\title{
Spatial Interference Mitigation for Multiple Input Multiple Output Ad Hoc Networks: MISO Gains
}

Salam Akoum, Marios Kountouris, Merouane Debbah, Heath Robert W.

\section{To cite this version:}

Salam Akoum, Marios Kountouris, Merouane Debbah, Heath Robert W.. Spatial Interference Mitigation for Multiple Input Multiple Output Ad Hoc Networks: MISO Gains. 2011 Conference Record of the Forty Fifth Asilomar Conference on Signals, Systems and Computers (ASILOMAR), Nov 2011, Pacific Grove, United States. 10.1109/ACSSC.2011.6190095 . hal-00648520

\section{HAL Id: hal-00648520}

https://hal-centralesupelec.archives-ouvertes.fr/hal-00648520

Submitted on 5 Dec 2011

HAL is a multi-disciplinary open access archive for the deposit and dissemination of scientific research documents, whether they are published or not. The documents may come from teaching and research institutions in France or abroad, or from public or private research centers.
L'archive ouverte pluridisciplinaire HAL, est destinée au dépôt et à la diffusion de documents scientifiques de niveau recherche, publiés ou non, émanant des établissements d'enseignement et de recherche français ou étrangers, des laboratoires publics ou privés. 


\title{
Spatial Interference Mitigation for Multiple Input Multiple Output Ad Hoc Networks: MISO Gains
}

\author{
Salam Akoum *, Marios Kountouris ${ }^{\dagger}$, Mérouane Debbah ${ }^{\ddagger}$, and Robert W. Heath, Jr. * \\ *Department of Electrical and Computer Engineering, The University of Texas at Austin, Austin, TX 78712-0240 \\ ${ }^{\dagger}$ Department of Telecommunications, Supélec, 91192 Gif-sur-Yvette, France \\ $\ddagger$ Alcatel-Lucent Chair on Flexible Radio, Supélec, 91192 Gif-sur-Yvette, France \\ \{salam.akoum,rheath\}@mail.utexas.edu, \\ \{marios.kountouris, merouane.debbah\}@ supelec.fr
}

\begin{abstract}
We consider spatial interference mitigation at the transmitter for multiple input single output ad hoc networks. We apply zero forcing beamforming at the transmitter, and analyze the corresponding network throughput and transmission capacity. Assuming a network with Poisson distributed transmitting nodes and spatially independent Rayleigh fading channels, we apply mathematical tools from stochastic geometry to derive a lower bound on the probability of outage. We derive scaling laws for the transmission capacity and show that for a large number of antennas, the maximum density of concurrently transmitting nodes scales linearly with the number of antennas at the transmitter, for a given outage constraint. Numerical results show that the network throughput achieved by interference nulling at the transmitter is comparable to that achieved by interference cancellation at the receiver.
\end{abstract}

\section{INTRODUCTION}

Mutual interference poses a major bottleneck on the throughput performance of mobile ad hoc networks. Employing multiple antennas at the radio nodes is a promising technique to mitigate interference and increase the network throughput. In particular, multiple antenna processing techniques at the receiver have been shown to yield linear performance in terms of maximum density scaling [1]. The role of multiple antennas in interference nulling at the transmitter, however, have not yet been fully investigated. This paper analyzes the gains from using multiple antennas for transmission in an ad hoc network. It applies transmit zero forcing beamforming for a multiple input single output (MISO) ad hoc network to increase the density of successful communication links.

Multiple antenna techniques for both single-user and multiuser multiple systems have been investigated in the literature [1]-[9] of mobile ad hoc networks. Single antenna transmission with multiple antennas interference cancellation at the

This work is funded in part by NSF: NSF-CCF-0830615 and by the DARPA ITMANET program under grant W911NF-07-1-0028. It is funded in part by the Agence Nationale de la Recherche within the project ANR-09VERSO: ECOSCELLS. M. Kountouris work is partially supported by project HIATUS. The project HIATUS acknowledges the financial support of the Future and Emerging Technologies (FET) programme within the Seventh Framework Programme for Research of the European Commission under FET-Open grant number: 265578. receiver was shown in [1] to achieve linear scaling of the maximum density of transmitting nodes with the number of antennas at the receiver. Maximum ratio transmission and zero forcing beamforming towards the strongest interferers at the receivers was analyzed in [3]. Outage probability and density scaling results for maximum ratio transmission and maximum ratio combining was reported in [6]. Open loop transmission with spatial multiplexing and orthogonal space time block codes and linear receivers was analyzed in [4]. The performance of multi-user MIMO communication in a Poisson field of interferers, with full and partial CSI at the transmitter was investigated in [7], [8]; it was shown that single-user transmission is optimal for most practical scenarios of interest. Multiple antenna interference nulling was considered in [9] for a two-tier spectrum sharing ad hoc network. Full zero forcing from the secondary network towards the primary receivers was applied at the transmitter. Using multiple antennas at the transmitter for same-tier interference mitigation was, however, not investigated. Prior work on spatial interference mitigation for same-tier mobile ad hoc networks [1]-[4] focused exclusively on multiple antenna processing at the receiver. Multiple antennas at the transmitter, if present, were used for maximum ratio transmission or open loop spatial loop multiplexing towards the associated receiver. The role of multiple antennas in interference mitigation at the transmitter was not considered.

Assuming the transmitter locations are distributed according to a homogeneous Poisson point process (PPP), we focus in this paper on devising a strategy to use multiple antennas for interference mitigation at the transmitter, to maximize the network throughput and increase the density of concurrently transmitting nodes. We consider a mobile ad hoc network with single-stream data links and single receive antennas. We apply zero forcing (ZF) to null the interference from the transmitters at the closest receivers. We derive bounds on the resulting probability of outage and the network throughput of the mobile ad hoc network. We derive the scaling law of the transmission capacity with the number of transmit antennas, for sufficiently large number of antennas, and a target outage constraint. In particular, we show that the maximum density 
of concurrently transmitting nodes in the network scales linearly with the number of antennas at the transmitter, as the number of antennas increases.

\section{System Model And Performance Metrics}

Consider an ad hoc network in which the transmitting nodes are located according to a two-dimensional homogeneous PPP $\Phi_{0}$ with intensity $\lambda_{0}$ transmitters per unit area. Each transmitter attempts to transmit with a probability $P_{a}$, independently of other transmitters, following a slotted ALOHA random access protocol. The process of active transmitters in each slot also forms a homogeneous PPP $\Phi$ with intensity $\lambda=\lambda_{0} P_{a}$. The location of the $n$-th active transmitter is denoted by $T_{n}$. Each transmitter communicates with a receiver at a fixed distance $d$ away from it. The receivers are not part of the transmitter PPP. The location of the $n$-th receiver is denoted by $R_{n}$.

By the stationarity of the Poisson process, we consider the performance of a typical transmitter-receiver link, $T_{0^{-}}$ $R_{0}$. From the perspective of $R_{0}$ and by Slivnyak's theorem [10], the process of interfering transmitters also forms a homogeneous PPP of intensity $\lambda$. The transmitting nodes in the network are equipped with $N$ antennas; whereas the receivers have a single antenna. The channel corresponding to the desired signal between $T_{0}$ and $R_{0}$ is denoted by $\mathbf{h}_{0} \in \mathbb{C}^{N \times 1}$. The interfering channel between $R_{0}$ and the $n$-th transmitting node $T_{n}$ is denoted by $\mathbf{g}_{n} \in \mathbb{C}^{N \times 1}$. The symbol transmitted from $T_{n}$ for $R_{n}$ is given by $s_{n}$, such that $\mathbb{E}\left[\left|s_{n}\right|^{2}\right]$ is normalized to one, for all $n$. We use the simplified attenuation model for the distance-dependent pathloss, $\ell\left(\mathrm{d}_{n}\right)=\gamma \mathrm{d}_{n}^{-\alpha}$, where $\gamma$ is the fixed transmit power at $T_{n}$ and $d_{n}$ is the distance between $T_{n}$ and $R_{0}$. The pathloss exponent is denoted by $\alpha>2$. The received signal at the tagged receiver $R_{0}$ for a frequency flat channel is given by

$$
\begin{aligned}
y_{0} & =\sqrt{\gamma} \mathrm{d}^{-\frac{\alpha}{2}} \mathbf{h}_{0}^{*} \mathbf{f}_{0} s_{0} \\
& +\sqrt{\gamma} \sum_{T_{n} \in \Phi} \mathrm{d}_{n}^{-\frac{\alpha}{2}} \mathbf{g}_{n}^{*} \mathbf{w}_{n} r_{n}+v_{0}
\end{aligned}
$$

where the vector $\mathbf{f}_{0} \in \mathbb{C}^{N \times 1}$ is the beamforming vector at $T_{0}, \mathbf{w}_{n} \in \mathbb{C}^{N \times 1}$ is the beamforming vector at $T_{n}$ for all $n$, and $v_{0} \in \mathbb{C}$ is the additive white Gaussian noise at $R_{0}$ with variance $\sigma^{2}$. We assume that the elements of the channel matrices $\mathbf{h}_{0}$ and $\mathbf{g}_{n}$ are independent and identically distributed $\mathcal{C N}(0,1)$, modeling a richly scattered independent fading environment.

We assume that each transmitter has knowledge of the channel to its intended receiver, and the channels to the adjacent receivers that the transmitter is interfering with. The CSI assumptions in this paper are applicable for time division duplexing (TDD) systems. The same assumptions on the knowledge of the channels at the receiving nodes have been considered in [1], [2], [8].

A primary performance metric of interest in this paper is the probability of outage $P_{\text {out }}$ with respect to a predefined signal-to-interference-plus-noise ratio (SINR) $\beta$,

$$
P_{\text {out }}=\mathbb{P}(\operatorname{SINR} \leq \beta) .
$$

The SINR statistics at $R_{0}$ depend on the design of the beamforming vectors $\mathbf{f}_{0}$ and $\mathbf{w}_{n}$, for all $n$. The probability of outage is used to compute the network throughput. The network throughput is defined as the product of the sum rate per unit area and the probability of success assuming that capacity achieving codes are used. It is given by

$$
T=\lambda \log _{2}(1+\beta) \mathbb{P}(\operatorname{SINR}>\beta) .
$$

The network throughput is different from the transmission capacity which is defined as the area spectral efficiency for the maximum density $\lambda_{\epsilon}=\sup \{\lambda: \mathbb{P}(\operatorname{SINR} \leq \beta) \leq \epsilon\}$ allowed subject to an outage constraint $\epsilon>0$, i.e.,

$$
C=\lambda_{\epsilon} \log _{2}(1+\beta)(1-\epsilon) .
$$

In contrast to the network throughput that may result in high outage events, the transmission capacity calculates the maximum density of transmissions per unit area so that a desired outage level $\epsilon$ is not exceeded.

\section{INTERFERENCE NULLING AT THE TRANSMITTER}

We propose a transmission strategy based on $\mathrm{ZF}$ interference nulling at the transmitter. We assume that each transmitter employs a partial ZF (PZF) beamforming vector. PZF was first suggested in [1] for receive filter design. It was found to be amenable to analysis and to explicitly balance interference cancellation and boosting of the desired signal power. With PZF, the transmitter uses $\ell$ degrees of freedom to null its interference to its closest, in terms of distance, receivers, and uses the remaining $N-\ell$ degrees of freedom to transmit the desired signal to its associated receiver. With no interference nulling $\ell=0, \mathrm{PZF}$ is equivalent to transmit beamforming; whereas for $\ell=N-1$ it is equivalent to full zero forcing beamforming.

To illustrate PZF beamforming, let

$$
\mathbf{H}_{0}=\left[\begin{array}{llll}
\mathbf{h}_{1} & \mathbf{h}_{2} & \cdots & \mathbf{h}_{\ell}
\end{array}\right], \quad \ell \leq N-1
$$

be a $N \times \ell$ matrix of channels from $T_{0}$ to its closest receivers. The vectors $\mathbf{h}_{1}, \cdots, \mathbf{h}_{\ell}$ are such that $\mathrm{d}_{01}<\mathrm{d}_{02}<\cdots<\mathrm{d}_{0 \ell}$ where $\mathrm{d}_{0 n}$ is the distance from $T_{0}$ to receiver $R_{n}$. The PZF beamforming vector $f_{0}$ is chosen such that it is in the null space of $\mathbf{H}_{0}, N u l l\left(\mathbf{H}_{0}\right)$, of dimension $N-\ell$. Furthermore, to maximize the desired signal power, $\mathbf{f}_{0}$ is taken in the direction of the projection of the desired channel $\mathbf{h}_{0}$ onto $\operatorname{Null}\left(\mathbf{H}_{0}\right)$. More precisely, $\mathbf{f}_{0}$ is computed as

$$
\mathbf{f}_{0}=\mathbf{Q Q}^{*} \mathbf{h}_{0}
$$

where $\mathbf{Q}$ forms an orthonormal basis for $\operatorname{Null}\left(\mathbf{H}_{0}\right)$.

For the proposed spatial interference strategy, the signalto-interference-plus-noise ratio (SINR) at the tagged receiver 


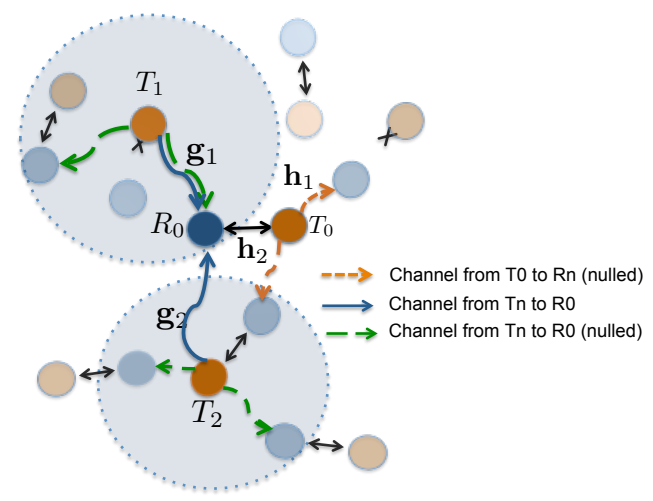

Fig. 1. Partial zero forcing interference nulling at the transmitter. The interference at the tagged receiver $R_{0}$ is nulled by its closest interferer $T_{1}$ but not nulled by its second interferer $T_{2}$. The cardinality and the location of the cancelled interferers in the set $\left\{C_{t}\right\}$ in the aggregate interference at $R_{0}$ is unknown.

$R_{0}$ is written as

$\operatorname{SINR}=\frac{\gamma \mathrm{d}^{-\alpha}\left|\mathbf{h}_{0}^{*} \mathbf{f}_{0}\right|^{2}}{\sigma^{2}+\gamma \sum_{T_{n} \in \Phi /\left\{C_{t}\right\}} \mathrm{d}_{n}^{-\alpha}\left|\mathbf{g}_{n}^{*} \mathbf{w}_{n}\right|^{2}}=\frac{\left|\mathbf{h}_{0}^{*} \mathbf{f}_{0}\right|^{2}}{\frac{1}{\mathrm{SNR}}+\mathrm{d}^{\alpha} I_{t}}$,

where SNR $=\frac{\gamma \mathrm{d}^{-\alpha}}{\sigma^{2}}$ is the signal-to-noise ratio at $R_{0}$. The set $\left\{C_{t}\right\}$ is the set of the transmitters that nulled their interference towards $R_{0}$. The cardinality $\left|C_{t}\right|$ of $\left\{C_{t}\right\}$ as well as the location of the nodes in $\left\{C_{t}\right\}$ with respect to $R_{0}$, are unknown. They depend on the density of the nodes $\lambda$ in the network and the distance from the transmitters to the receiver $R_{0}$. For transmit PZF, interference nulling is done "blindly" towards the closest receivers to the transmitter. The aggregate interference at the tagged receiver $R_{0}$ is not taken into account in the nulling performed at $T_{0}$. Consequently, the aggregate interference at $R_{0}$ need not be void of the closest $\ell$ interferers to $R_{0}$ as in the case of PZF receiver cancellation [1]. This is illustrated in Figure 1 , where $T_{1}$ and $T_{2}$ are the closest interferers to $R_{0}$, the interference from $T_{1}$ is removed at $R_{0}$ while that of $T_{2}$ remains.

The desired signal power $\left|\mathbf{h}_{0}^{*} \mathbf{f}_{0}\right|^{2}$ is Chi-squared distributed with $2(N-\ell)$ degrees of freedom, $\chi_{2(N-\ell)}^{2}$. This follows from the projection of a random vector $\mathbf{h}_{0}$ onto an independent $(N-\ell)$-dimensional space $\operatorname{Null}\left(\mathbf{H}_{0}\right)$. The distribution of the aggregate interference term depends on the characterization of the set $\left\{C_{t}\right\}$. We compute an upper bound on the expected value of the interference in the following Lemma.

Lemma 1: The expected interference power $I_{t}$ at $R_{0}$ is given by

$$
\mathbb{E}\left[I_{t}\right]=\left(\pi \mathrm{d}^{2} \lambda_{p}\right)^{\frac{\alpha}{2}}\left(\frac{\alpha}{2}\left(\frac{\alpha}{2}-1\right)^{-1}\right),
$$

where $\lambda_{p}=\lambda-\rho \ell$, and $\rho$ is the inverse of the area occupied by the interferers on the two-dimensional network space.

Proof: The proof is provided in Appendix A.

The upper bound on $\mathbb{E}\left[I_{t}\right]$ can be understood by arguing that on average each receive node in the network receives interference from all the transmitters except $\ell$ interferers, chosen at random from the total set of interferers $\Phi$. This implies that the density of interferers is reduced by $\rho \ell$ where $\rho$ is the inverse of the area occupied by the network. The value of $\rho$ does not affect the conclusions drawn on the scaling performance of the network.

Using the distribution of the desired signal power, and the upper bound on the expected interference term, we find an upper bound on the probability of outage for transmit PZF.

Theorem 1: The probability of outage for transmit $\mathrm{ZF}$ beamforming for an ad hoc network with PPP distributed nodes with density $\lambda$ and $N$ transmit antennas is upper bounded by

$$
P_{\text {out }}^{\mathrm{T}-\mathrm{PZF}} \leq \frac{\beta\left(\frac{\alpha}{2}\left(\frac{\alpha}{2}-1\right)^{-1}\left(\pi \mathrm{d}^{2}(\lambda-\rho \ell)\right)^{\frac{\alpha}{2}}+\frac{1}{\mathrm{SNR}}\right)}{N-\ell-1} .
$$

Proof: We apply the Markov inequality on the tail probability of the inverse SINR to get

$$
\begin{gathered}
\mathbb{P}[\operatorname{SINR} \leq \beta]=\mathbb{P}\left[\frac{1}{\operatorname{SINR}} \geq \frac{1}{\beta}\right] \leq \beta \mathbb{E}\left[\frac{1}{\operatorname{SINR}}\right] \\
\stackrel{(a)}{=} \beta \mathbb{E}\left[\frac{1}{\left|\mathbf{h}_{0}^{*} \mathbf{f}_{0}\right|^{2}}\right]\left[\mathbb{E}\left[\frac{1}{\operatorname{SNR}}\right]+\mathbb{E}\left[\mathrm{d}^{\alpha} I_{t}\right]\right] \\
\leq \frac{\beta\left(\frac{\alpha}{2}\left(\frac{\alpha}{2}-1\right)^{-1}\left(\pi \mathrm{d}^{2} \lambda_{p}\right)^{\frac{\alpha}{2}}+\frac{1}{\operatorname{SNR}}\right)}{N-\ell-1}
\end{gathered}
$$

The expression in (a) is obtained because the signal and interference plus noise terms are independent. The expected value of the desired signal power is given by $N-\ell-1$. The upper bound on the probability of outage is finally computed using the upper bound from Lemma 1.

A bound on the network throughput $T$ is obtained from the complement of the probability of outage as follows

$$
T \geq\left[1-\frac{\beta\left(\frac{\alpha}{2}\left(\frac{\alpha}{2}-1\right)^{-1}\left(\pi \mathrm{d}^{2}(\lambda-\rho \ell)\right)^{\frac{\alpha}{2}}+\frac{1}{\mathrm{SNR}}\right)}{N-\ell-1}\right]
$$

The transmission capacity $C$ is a function of the maximum density $\lambda_{\epsilon}$ such that the probability of outage is less than a threshold $\epsilon$. A lower bound on the maximum density is obtained by setting the probability of outage expression in Theorem 1 equal to $\epsilon$ and solving for $\lambda$. It is given by

$$
\lambda_{\epsilon} \geq\left(\frac{(N-\ell-1) \epsilon-\frac{\beta}{\mathrm{SNR}}}{\beta\left(\pi \mathrm{d}^{2}\right)^{\frac{\alpha}{2}} \frac{\alpha}{2}\left(\frac{\alpha}{2}-1\right)^{-1}}\right)^{\frac{2}{\alpha}}+\rho \ell .
$$

In what follows, we use the bound on the transmission capacity to derive the main result on the scaling of the transmit PZF beamforming system with the number of transmit antennas. 


\section{LINEAR DENSITY SCALING}

To establish the scaling of the density $\lambda_{\epsilon}$ with the number of transmit antennas $N$ for transmit PZF beamforming, we choose the number of nulled receivers $\ell$ to be a constant fraction of the total number of antennas

$$
\ell=\theta N, \quad 0<\theta<1 .
$$

We rewrite the lower bound on the density $\lambda_{\epsilon}$ in terms of $\theta$ as

$$
\begin{gathered}
\lambda_{\epsilon} \geq\left(\left(\frac{\epsilon}{\beta}\right)^{\frac{2}{\alpha}} \frac{\left(\frac{\alpha}{2}-1\right)^{\frac{2}{\alpha}}\left(\frac{2}{\alpha}\right)^{\frac{2}{\alpha}}}{\pi \mathrm{d}^{2}}(1-\theta)^{\frac{2}{\alpha}}\left(1-\frac{1+\frac{\beta}{\mathrm{SNR}}}{(1-\theta) N}\right)^{\frac{2}{\alpha}}\right. \\
\left.+\rho \theta N^{1-\frac{2}{\alpha}}\right) N^{\frac{2}{\alpha}} .
\end{gathered}
$$

As the number of antennas $N$ increases, for sufficiently large $N$, the lower bound on $\lambda_{\epsilon}$ scales linearly with $N$. More formally, as $N \rightarrow \infty$,

$$
\frac{\lambda_{\epsilon}}{N} \geq \rho \theta
$$

This shows linear scaling of the density $\lambda_{\epsilon}$ with the number of transmit antennas when transmit ZF beamforming is employed. The linear scaling result can be understood by considering Lemma 1, as on average the aggregate interference power at $R_{0}$ is reduced by $\ell=\theta N$. This provides a linear scaling of the interference term with $N$. Similarly, the desired signal power increases linearly with $N$. The signal and interference terms both increase with the same scaling with $N$ and thus the linear scaling is maintained for sufficiently large $N$.

\section{Simulation Results And Discussion}

In this section, we present Monte Carlo simulations to evaluate the performance of the transmit ZF scheme proposed in the paper. The simulation setup follows that in [3]. The distance between each transmitter and its associated receiver is $\mathrm{d}=10 \mathrm{~m}$, and the pathloss exponent is set to $\alpha=4$. The probability of outage threshold $\beta$ is taken equal to 3 corresponding to an SINR threshold value of $4.8 \mathrm{~dB}$.

Figure 2 plots the density $\lambda_{\epsilon}$ of transmitting nodes on a $\log -\log$ scale for increasing number of transmit antennas $N$, for $\epsilon=0.1$. It compares the numerically computed density, with the lower bound obtained in Theorem 1, and the lower bound obtained in [1] for ZF interference cancellation at the receiver for a single input multiple output (SIMO) system, with $N$ receive antennas. We observe from the figure that the bounds and the numerically computed density indeed show a linear scaling of $\lambda_{\epsilon}$ with $N$. The lower bound of Theorem 1 is tighter for a small number of antennas. The plot also suggests that the simulated transmit ZF beamforming density scaling approaches the bound for the SIMO interference cancellation scheme, as the number of antennas increases.

In Figure 3, the network throughput is plotted for an increasing number of antennas $N$, and an outage probability

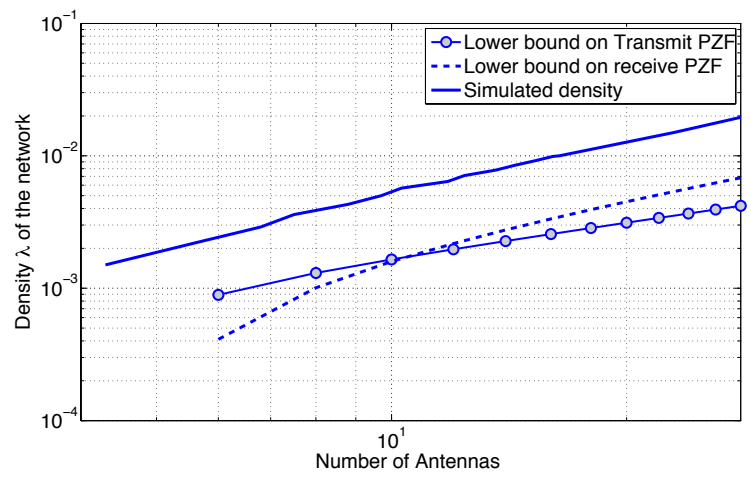

Fig. 2. The maximum density of transmitting nodes $\lambda_{\epsilon}$ versus the number of transmit antennas $N$. The simulated density refers to the density scaling obtained by Monte Carlo simulations for $\epsilon=0.1$. The analytical transmit nulling density refers to the density obtained in Theorem 1 . The receiver cancelation density is obtained in [1]

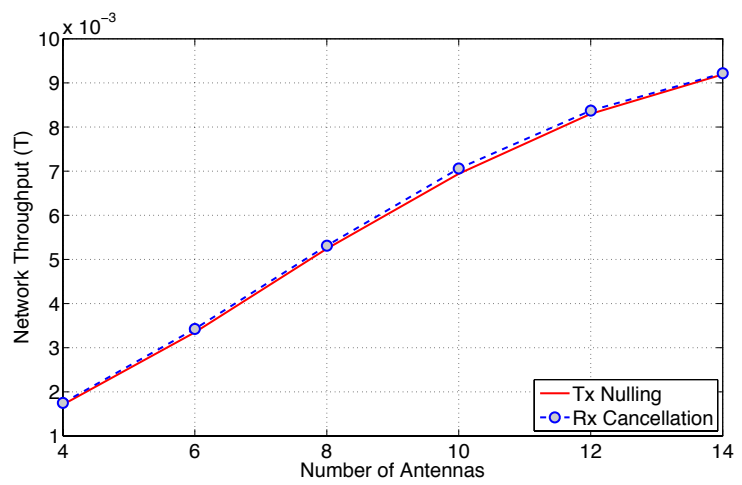

Fig. 3. The network throughput as a function of the number of antennas $N$ for MISO ZF transmit nulling and SIMO ZF receive cancellation in [1].

target $\epsilon=0.1$. The network throughput achieved using transmit ZF beamforming is compared with that of the SIMO interference cancellation scheme in [1]. Figure 3 shows that the two network throughputs are almost equivalent. This clearly illustrates that receive processing techniques, while achieving good performance, should not be considered in exclusivity when it comes to interference reduction in multiple antenna ad hoc networks. Transmit processing using a "blind" interference nulling strategy yields good performance, and removes the processing burden from the receiver.

Finally, although linear density scaling is shown to be achieved for any $0<\theta<1$ for transmit PZF, we can find the optimal fraction of antennas $\theta$ that should be used at the transmitter to null the interference, while maximizing the density of concurrent transmitters in the network. Figure 4 plots $\lambda_{\epsilon}$ versus the number of nulled interferers $\ell$ for $N=10$ and $\epsilon=0.1$. The maximum density is shown to be achieved at $\ell=5$ for $\alpha=4$. This agrees with the optimal fraction $\theta^{*}=1-\frac{2}{\alpha}$ obtained for ZF interference cancellation in [1]. 


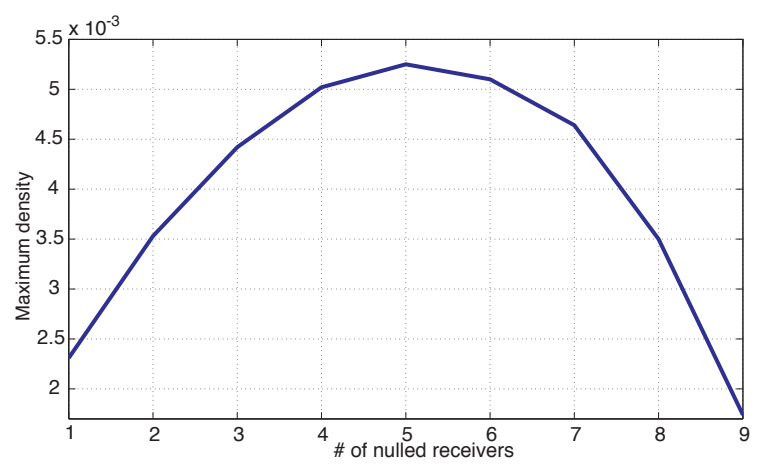

Fig. 4. The maximum density $\lambda_{\epsilon}$ versus the number of nulled receivers $\ell$ for $N=10, \epsilon=0.1$ and $\alpha=4$. The maximum is reached at $\ell=5$.

\section{CONCLUSION}

In this paper, we analyzed spatial interference mitigation for a MISO ad hoc network. We showed that ZF interference nulling at the transmitter provides a linear increase in the transmission capacity with the number of antennas at the transmitter, for a given outage constraint. Although transmit PZF is applied towards the closest receivers, which do not necessarily correspond to the closest interferers to the desired receiver, its network throughput performance is comparable to the performance of SIMO ZF cancellation, where cancellation is done deterministically towards the closest interferers at the receiver. The main takeaway from this paper is that multiple antennas at the transmitter should be used more efficiently to improve the throughput performance of mobile ad hoc networks. Future work includes devising a distributed multiple input multiple output interference mitigation strategy, where in addition to interference nulling at the transmitter, antennas at the receiver are used to cancel residual interference.

\section{APPENDIX A \\ PROOF OF LEMMA 1} by

The expected value of the aggregate interference $I_{t}$ is given

$$
\begin{aligned}
\mathbb{E}\left[\sum_{T_{n} /\left\{C_{t}\right\}} \mathrm{d}_{n}^{-\alpha}\left|\mathbf{g}_{n}^{*} \mathbf{w}_{n}\right|^{2}\right] & =\sum_{T_{n} /\left\{C_{t}\right\}} \mathbb{E}\left[\mathrm{d}_{n}^{-\alpha}\left|\mathbf{g}_{n}^{*} \mathbf{w}_{n}\right|^{2}\right] \\
& =\sum_{T_{n} /\left\{C_{t}\right\}} \mathbb{E}\left[\mathrm{d}_{n}^{-\alpha}\right]
\end{aligned}
$$

The distances $\mathrm{d}_{1}^{2}, \mathrm{~d}_{2}^{2}, \cdots$ form a one dimensional PPP with intensity $\pi \lambda_{p}$. The density $\lambda_{p}$ is written as a function of $\lambda$ as follows

$$
\lambda_{p}=\lambda-\rho \ell,
$$

where $\rho$ is the inverse of the area occupied by the interferers, or the two dimensional space of the ad hoc network. The random variables $\pi \lambda_{p} \mathrm{~d}_{n}^{2}$ are distributed as $\chi_{2 n}^{2}$, and thus have
PDF $f(x)=\frac{x^{n-1} e^{-x}}{(n-1) !}$. Therefore

$$
\mathbb{E}\left[\mathrm{d}_{n}^{-\alpha / 2}\right]=\left(\pi \lambda_{p}\right)^{\frac{\alpha}{2}} \frac{\Gamma(n-\alpha / 2)}{\Gamma(n)} .
$$

This quantity is finite only for $n>\frac{\alpha}{2}$, and thus the expected power from the nearest uncancelled interferer is finite only if $n>\frac{\alpha}{2}$. The upper bound on the expected interference, for $n \geq\left\lceil\frac{\alpha}{2}\right\rceil$, is given by,

$$
\begin{aligned}
\mathbb{E}\left[I_{t}\right] & =\left(\pi \lambda_{p}\right)^{\frac{\alpha}{2}} \sum_{n=\left\lceil\frac{\alpha}{2}\right\rceil}^{\infty} \frac{\Gamma\left(n-\frac{\alpha}{2}\right)}{\Gamma(n)} \\
& \stackrel{(a)}{<}\left(\pi \lambda_{p}\right)^{\alpha / 2}\left(1+\sum_{n=\left\lceil\frac{\alpha}{2}\right\rceil+2}^{\infty}\left(n-\left\lceil\frac{\alpha}{2}\right\rceil\right)^{-\frac{\alpha}{2}}\right) \\
& \stackrel{(b)}{\leq} \quad\left(\pi \lambda_{p}\right)^{\frac{\alpha}{2}}\left(1+\int_{\left\lceil\frac{\alpha}{2}\right\rceil+1}^{\infty}\left(x-\left\lceil\frac{\alpha}{2}\right\rceil\right)^{-\frac{\alpha}{2}} d x\right) \\
& =\left(\pi \lambda_{p}\right)^{\frac{\alpha}{2}}\left(\frac{\alpha}{2}\left(\frac{\alpha}{2}-1\right)^{-1}\right)
\end{aligned}
$$

where (a) is reached by invoking Kershaw's inequality on the Gamma function [3],

$$
\frac{\Gamma(i-\alpha / 2)}{\Gamma(i)}<\left(i-\left\lceil\frac{\alpha}{2}\right\rceil\right)^{-\frac{\alpha}{2}},
$$

and (b) is computed by upper bounding the summation by the integration.

\section{REFERENCES}

[1] N. Jindal, J. G. Andrews, and S. Weber, "Multi-antenna communication in ad hoc networks: achieving MIMO gains with SIMO transmission," IEEE Trans. Commun., vol. 59, pp. 529-540, Feb. 2011.

[2] R. Vaze and R. W. Heath Jr., "Transmission capacity of adhoc networks with multiple antennas using transmit stream adaptation and interference cancelation," to appear in IEEE Trans. Inform. Theory, 2011. [Online]. Available: ArXiv preprint at http://arxiv.org/abs/0912.2630

[3] K. Huang, J. G. Andrews, D. Guo, R. W. Heath Jr., and R. Berry, "Spatial interference cancellation for multi-antenna mobile ad hoc networks," to appear in IEEE Trans. Inform. Theory, 2011. [Online]. Available: Arxiv preprint at http://arxiv.org/abs/0807.1773

[4] R. Louie, M. McKay, and I. Collings, "Open-loop spatial multiplexing and diversity communications in ad hoc networks," IEEE Trans. Inform. Theory, vol. 57, no. 1, pp. 317 - 344, Jan. 2011.

[5] O. B. S. Ali, C. Cardinal, and F. Gagnon, "Performance of optimum combining in a poisson field of interferers and rayleigh fading channels," IEEE Trans. Wireless Commun., vol. 9, no. 8, p. 24612467, Aug. 2010.

[6] A. Hunter, J. G. Andrews, and S. Weber, "The transmission capacity of ad hoc networks with spatial diversity," IEEE Trans. Wireless Commun., vol. 7, no. 12, pp. 5058 - 5071, Dec. 2008.

[7] M. Kountouris and J. G. Andrews, "Capacity bounds on multiuser MIMO transmission in random wireless networks," submitted to IEEE Trans. Inform. Theory, 2010.

[8] - "Downlink SDMA with limited feedback in interferencelimited wireless networks," submitted to IEEE Trans. Wireless Commun., 2011. [Online]. Available: ArXiv preprint at http://arxiv.org/abs/1103.0561

[9] R. Vaze, "Transmission capacity of spectrum sharing ad-hoc networks with multiple antennas," IEEE Trans. Wireless Commun., vol. 10, no. 7 , pp. 2334 - 2340, Jul. 2011.

[10] F. Baccelli and B. Blaszczyszyn, Stochastic geometry and wireless networks. Volume I: theory. NOW publishers, 2009. 